\title{
Determining Exchange Splitting in a Magnetic Semiconductor by Spin-Filter Tunneling
}

\author{
T. S. Santos,${ }^{1}$ J. S. Moodera, ${ }^{1}$ K. Venkataraman,${ }^{1}$ E. Negusse,${ }^{2}$ \\ J. Holroyd ${ }^{2}$ J. Dvorak,${ }^{2}$ M. Liberati, ${ }^{2}$ Y. U. Idzerda, ${ }^{2}$ and E. Arenholz ${ }^{3}$ \\ ${ }^{1}$ Francis Bitter Magnet Laboratory, \\ Massachusetts Institute of Technology, Cambridge, Massachusetts 02139 \\ ${ }^{2}$ Department of Physics, Montana State University, Bozeman, Montana 59717 \\ ${ }^{3}$ Advanced Light Source, Lawrence Berkeley National Laboratory, Berkeley, California 94720
}

(Dated: June 19, 2008)

\begin{abstract}
A large exchange splitting of the conduction band in ultrathin films of the ferromagnetic semiconductor $\mathrm{EuO}$ was determined quantitatively, by using EuO as a tunnel barrier and fitting the current-voltage characteristics and temperature dependence to tunneling theory. This exchange splitting leads to different tunnel barrier heights for spin-up and spin-down electrons, and is large enough to produce a near fully spin-polarized current. Moreover, the magnetic properties of these ultrathin films $(<6 \mathrm{~nm})$ show a reduction in Curie temperature with decreasing thickness, in agreement with theoretical calculation [R. Schiller et al., Phys. Rev. Lett. 86, 3847 (2001)].
\end{abstract}

PACS numbers: $\mathrm{xxx}$ 
Ferromagnetic semiconductors (FSs) having a tunable charge carrier concentration that is spin-polarized are ideally suited as spin injectors and detectors in semiconductor spintronic devices. The europium chalcogenides stand out among the FSs as ideal Heisenberg ferromagnets, with a high magnetic moment and a large exchange splitting of the conduction band [1]. Utilizing the exchange splitting $\left(2 \Delta \mathrm{E}_{\mathrm{ex}}\right)$ to filter spins, these materials produce a near fully spin-polarized current when used as a tunnel barrier [2-4], making them strong candidates for spin injection in semiconductors. Of the Eu chalcogenides, EuO has the largest $2 \Delta \mathrm{E}_{\mathrm{ex}}$ and the highest Curie temperature $\left(\mathrm{T}_{\mathrm{C}}=69 \mathrm{~K}\right.$ for bulk). However, due to the high reactivity of $\mathrm{EuO}$ with air, preparation of thin films of $\mathrm{EuO}$ is highly challenging, precluding the study of EuO down to a few monolayers. Successful preparation of chemically-stable, high-quality EuO as tunnel barriers in this work has allowed for the study of EuO at the ultrathin thickness scale, illuminating its magnetic properties, exchange splitting and spin-filter capability at few monolayers range.

In $\mathrm{EuO}$ the large saturation magnetic moment $\mu g J=7 \mu_{B}$ per $\mathrm{Eu}^{2+}$ originates from the seven unpaired electrons localized at the $4 f$ levels in the energy gap between the valence band and conduction band, shown schematically in Fig. 1. The optical band gap, $\left(\mathrm{E}_{g}=1.1 \mathrm{eV}\right)$ is the energy gap between the $4 f$ levels and the conduction band. Ferromagnetic order of the $4 f$ spins causes exchange splitting of the conduction band, lowering (raising) the spin-up(down) band by $\Delta \mathrm{E}_{\mathrm{ex}}$. Thus, free carriers in the conduction band are spin-polarized. A large exchange splitting of $0.54 \mathrm{eV}$ was determined by measuring the red shift of the absorption edge in single crystals of EuO cooled below $\mathrm{T}_{\mathrm{C}}[1,5]$.

When ultrathin films of EuO are used as the tunnel barrier between two metallic electrodes, the exchange splitting of the conduction band gives rise to a lower barrier height for spin-up electrons $\Phi_{\uparrow}\left(=\Phi_{0}-\Delta \mathrm{E}_{\mathrm{ex}}\right)$ and a higher barrier height for spin-down electrons $\Phi_{\downarrow}$ $\left(=\Phi_{0}+\Delta \mathrm{E}_{\mathrm{ex}}\right)$, where $\Phi_{0}$ is the barrier height at $\mathrm{T}>\mathrm{T}_{\mathrm{C}}$. Because the tunnel current depends exponentially on the barrier height [6], the tunneling probability for spin-up electrons is much greater than for spin-down electrons, leading to a highly spin-polarized current-a phenomenon called the spin-filter effect [7]. Consequently, a larger $2 \Delta \mathrm{E}_{\mathrm{ex}}$ results in a higher spin-filter efficiency. Using a spin-filter tunnel barrier, as opposed to a ferromagnetic metal, is a unique approach to achieving a spin-polarized current with built-in advantages, such as interfacial band matching and a sharper interface. 


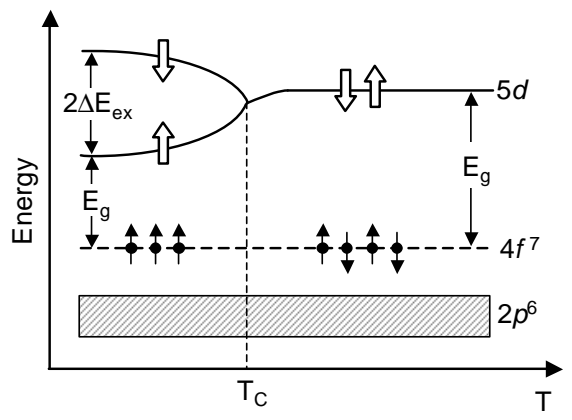

FIG. 1: Schematic of the energy gap of EuO, showing the exchange splitting in the $5 d$ conduction band for $\mathrm{T}<\mathrm{T}_{\mathrm{C}}$, lowering the spin-up sub-level from the spin-down sub-level by $2 \Delta \mathrm{E}_{\mathrm{ex}}$. Figure modified from Ref. [1].

Because the splitting in bulk EuO is significant compared to $\mathrm{E}_{g}$, EuO could potentially produce total spin polarization $(\mathrm{P}=100 \%)$. However, only a few monolayers of $\mathrm{EuO}$ is needed for tunneling, which can be expected to have different properties than bulk. For example, Schiller et al. [8] calculated the electronic structure and magnetic properties of ultrathin, single-crystalline, $\mathrm{EuO}(100)$ and found a reduction in $\mathrm{T}_{\mathrm{C}}$ from the bulk value as the thickness approached a few monolayers. However, due to the highly reactive nature of europium, and thus the difficulty in preparing ultrathin EuO films, their calculation had not been experimentally verified. Furthermore, a reduction in $\mathrm{T}_{\mathrm{C}}$ for ultrathin EuO raises the question as to whether a reduction in $2 \Delta \mathrm{E}_{\mathrm{ex}}$ should be expected as well, which is directly relevant to spin filtering.

In this study using well-characterized high-quality, ultrathin EuO films, we investigated this Heisenberg ferromagnet in the few-monolayers regime, allowing us to verify the model by Schiller et al. In addition, by detailed transport measurements of tunnel junctions with $\mathrm{EuO}$ barriers, we uniquely determined the amount of exchange splitting for ultrathin $\mathrm{EuO}$.

Films of EuO were made by thermal reactive deposition, whereby pure europium metal was evaporated in the presence of a dynamic oxygen partial pressure of $3 \times 10^{-7}$ Torr. $\mathrm{Eu}_{2} \mathrm{O}_{3}$, with a heat of formation $\Delta H_{f}=-1730 \mathrm{~kJ} / \mathrm{mol}$ compared to $-608 \mathrm{~kJ} / \mathrm{mol}$ for EuO [9], forms more readily. Because $\mathrm{Eu}_{2} \mathrm{O}_{3}$ is paramagnetic, and thus not a spin filter, the $\mathrm{Eu}$ metal evaporation rate and oxygen flow were carefully controlled in order to produce optimum EuO. To study the dependence of $\mathrm{T}_{\mathrm{C}}$ on film thickness, a wedge film was made 


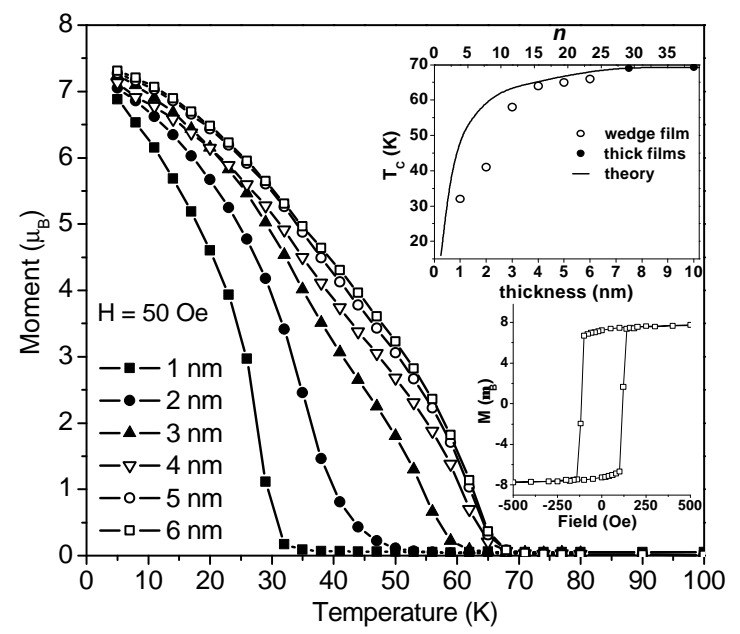

FIG. 2: Dependence of $\mathrm{T}_{\mathrm{C}}$ on thickness of $\mathrm{EuO}$ film with the structure: $2 \mathrm{~nm} \mathrm{Cr} / 9 \mathrm{~nm}$ $\mathrm{Cu} / \mathrm{EuO} / 2 \mathrm{~nm} \mathrm{Y/8} \mathrm{nm} \mathrm{Al}$. Top inset: $\mathrm{T}_{\mathrm{C}}$ dependence compared with Schiller et al. [8], where $n$ is the number of monolayers. The $\mathrm{T}_{\mathrm{C}}$ of thicker EuO films made separately are also plotted for reference. Bottom inset: Hysteresis loop of the $6 \mathrm{~nm}$ film at $5 \mathrm{~K}$. The measured moment $>7 \mu_{B}$ is likely due to an under-estimation of film thickness, as described in text.

with thickness ranging from $1 \mathrm{~nm}$ to $6 \mathrm{~nm}$, in steps of $1 \mathrm{~nm}$. The wedge was deposited by opening the shutter for each step, such that the top interface of the entire wedge film was the same. The magnetic and chemical properties of the films were found to be critically dependent upon the materials selection for the top and bottom interface layers $[4,10]$. The wedge was deposited onto a $\mathrm{Si} / 50 \mathrm{~nm} \mathrm{SiO}_{2}$ substrate with a $\mathrm{Cu}$ bottom layer and a Y capping layer. Films prepared under these conditions are known to be polycrystalline [4]. The films of various thicknesses were characterized by superconducting quantum interference device (SQUID) magnetometry, x-ray absorption spectroscopy (XAS) and magnetic circular dichroism (MCD). Tunnel junctions were patterned in situ using shadow masks, forming a cross configuration with a junction area of $(200 \times 200) \mu \mathrm{m}^{2}$. Deposition rate and thickness were determined in situ by a quartz crystal monitor. Given that the crystal is located at a distance from the substrate and the oxygen inlet, the actual thickness may be greater than the nominal thickness to within $15 \%$.

Figure 2 displays magnetization $(\mathrm{M})$ versus temperature (T) for 1-6 nm EuO films, showing a clear trend toward lower $\mathrm{T}_{\mathrm{C}}$ as film thickness decreases. The $\mathrm{T}_{\mathrm{C}}$ of the thicker films approach that of bulk, while the thinnest, $1 \mathrm{~nm}$ film has a $\mathrm{T}_{\mathrm{C}}$ of $30 \mathrm{~K}$. This is the general 
behavior of ferromagnetic films and is also what is predicted by the calculation of Schiller et al. This trend is caused by the lower coordination of the $\mathrm{Eu}^{2+}$ ions at the interfaces, such that the increasing, atomic surface-to-volume ratio for the thinner films leads to weaker exchange interactions. Experimental data is compared with Schiller et al.'s calculation in the inset of Fig. 2. Overall, the experimental values are lower than the theoretical curve. This is not unreasonable when considering that the calculation was done for free-floating, single-crystal $\mathrm{EuO}(100)$, whereas the films in this experiment are polycrystalline, with materials at the interfaces. The magnetic moment at $5 \mathrm{~K}$ closely matches the bulk value of $7 \mu_{B}$.

The chemical composition and magnetic properties of these ultrathin $\mathrm{EuO}$ films were further investigated using XAS and XMCD. At the europium $3 d_{5 / 2}\left(\mathrm{M}_{5}\right)$ absorption edge, $\mathrm{Eu}^{2+}$ and $\mathrm{Eu}^{3+}$ have distinct signature peaks, as shown in Fig. 3a for reference films of $\mathrm{EuO}$ and $\mathrm{Eu}_{2} \mathrm{O}_{3}$ [11-13]. For all film thicknesses the $\mathrm{Eu}^{2+}$ peak is clearly dominant, as shown in Fig. 3b. In order to obtain the relative amounts of $\mathrm{EuO}$ and $\mathrm{Eu}_{2} \mathrm{O}_{3}$, shown in the inset of Fig. 3b, the XAS spectra were deconvolved against the reference $\mathrm{EuO}$ and $\mathrm{Eu}_{2} \mathrm{O}_{3}$ spectra, resulting in $\sim 90 \% \mathrm{EuO}$ for all film thicknesses (see Fig. 3b inset).

The XMCD, measured at $18 \mathrm{~K}$ with $90 \%$ circularly-polarized light at $60^{\circ}$ incidence angle in the presence of a $\pm 0.5 \mathrm{~T}$ magnetic field, is presented in Fig. 3c as the difference in the $\mathrm{I}^{+}$and $\mathrm{I}^{-}$spectra, where $\mathrm{I}^{+}$and $\mathrm{I}^{-}$are the XAS spectra for parallel and antiparallel alignment of photon polarization and magnetization direction, respectively. The displayed XMCD are corrected for the fraction of $\mathrm{Eu}^{2+}$, incomplete light polarization, incidence angle, and finite temperature reduction of the moment (i.e. the spectra would be observed for pure $\mathrm{EuO}$ that is fully magnetized with $100 \%$ circular polarized light at grazing incidence and at $\mathrm{T}=0 \mathrm{~K})$. After the corrections, the maximum XMCD signal is measured to be $52.0 \% \pm 4.3 \%$. This is the highest XMCD signal reported for $\mathrm{EuO}$ and is in excellent agreement with the theoretically expected value of $51 \%$ for $\mathrm{EuO}[11,13]$. Additionally, the $\mathrm{Eu}^{2+}$ XAS and XMCD spectra are identical to the $\mathrm{Gd}^{3+}$ spectra for GdN as measured by Leuenberger [14], as was theoretically proposed [11]. For comparison, the normalized XMCD spectra for all thicknesses are shown in the inset of Fig. 3c, confirming that the dichroism, and hence the magnetization, originates entirely from $\mathrm{EuO}$, as expected. These XAS and XMCD measurements, along with the SQUID measurements, show the excellent quality of these ultrathin $\mathrm{EuO}$ films. 


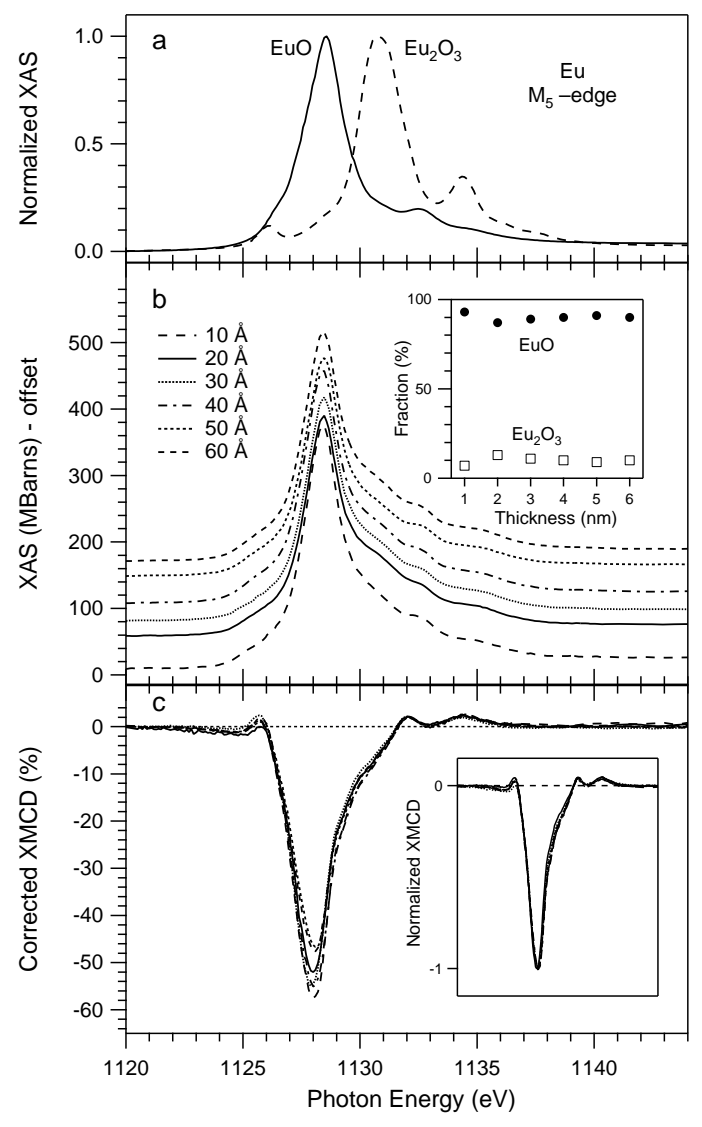

FIG. 3: a) Normalized reference XAS spectra at the $\mathrm{M}_{5}$ edge for an $\mathrm{EuO}$ and $\mathrm{Eu}_{2} \mathrm{O}_{3}$ film. b) XAS of the $1 \mathrm{~nm}-6 \mathrm{~nm} \mathrm{EuO}$ wedge (same wedge as in Fig. 2) at $295 \mathrm{~K}$. Inset: Relative amounts of EuO and $\mathrm{Eu}_{2} \mathrm{O}_{3}$ at each wedge step, computed by deconvolving the XAS spectra. c) Corrected XMCD spectra of the wedge; corrections as described in text. Inset: Normalized MCD spectra overlaid, confirming that the dichroism originates only from ferromagnetic EuO.

Next, we address the transport behavior. The junction resistance $\left(R_{J}\right)$ of tunnel junctions with the structure $3.6 \mathrm{~nm} \mathrm{Al} / 0.4 \mathrm{~nm} \mathrm{Mg} / 2.5 \mathrm{~nm} \mathrm{EuO/4.0} \mathrm{nm} \mathrm{Y/8.0} \mathrm{nm} \mathrm{Al} \mathrm{[15]} \mathrm{was} \mathrm{measured}$ as a function of $\mathrm{T}$, using a 4-point configuration with a constant bias applied with respect to the $\mathrm{Y}$ electrode. As $\mathrm{T}$ decreased below room temperature, $\mathrm{R}_{\mathrm{J}}$ increased as is common for a semiconducting barrier, whereas at low temperatures $R_{J}$ decreased. As shown in Fig. 4a, when the junction was cooled below the $\mathrm{T}_{\mathrm{C}}$ of $\mathrm{EuO}, \mathrm{R}_{\mathrm{J}}$ decreased dramatically. This is due to the lowering of the spin-up barrier height caused by the exchange splitting in EuO (see Fig. 1). The drop in $R_{J}$ is large, as much as two orders of magnitude for these junctions, showing substantial exchange splitting in these ultrathin $\mathrm{EuO}$ films. Such a resistance drop 

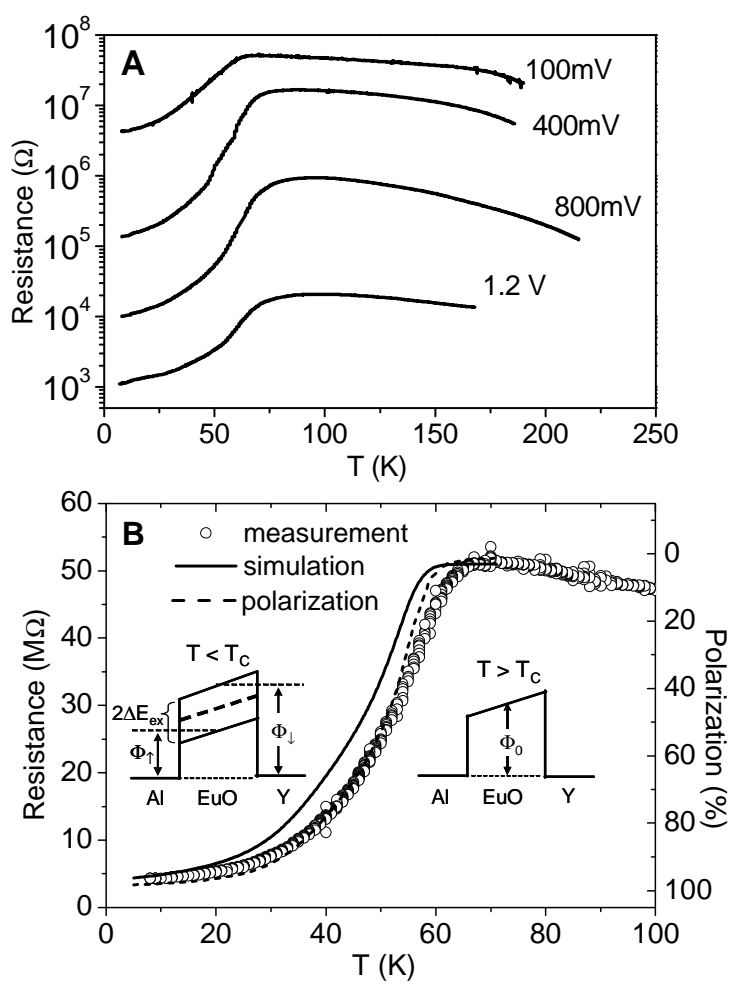

FIG. 4: a) $\mathrm{R}_{\mathrm{J}}(\mathrm{T})$ for a $\mathrm{Al} / \mathrm{EuO} / \mathrm{Y}$ tunnel junction, measured at the biases indicated, shows the reduction in $R_{J}$ for $T<T_{C}$ due to exchange splitting in the EuO barrier. b) $R_{J}(T)$ measured with $100 \mathrm{mV}$, replotted from (a) for clarity. $\mathrm{R}_{\mathrm{J}}(\mathrm{T})$ was simulated using the $\mathrm{M}(\mathrm{T})$ behavior for the $3 \mathrm{~nm}$ film from Fig. 2 and assuming that $\mathrm{M}(\mathrm{T}) \propto 2 \Delta \mathrm{E}_{\mathrm{ex}}(\mathrm{T})$. Zero $\mathrm{P}$ was deduced for $\mathrm{T}>\mathrm{T}_{\mathrm{C}}$, and $\mathrm{P}$ increased as splitting increased for $\mathrm{T}<\mathrm{T}_{\mathrm{C}}$, reaching $98 \%$. Inset: Energy barrier profile for $\mathrm{T}<\mathrm{T}_{\mathrm{C}}$ and $\mathrm{T}>\mathrm{T}_{\mathrm{C}}$.

was consistently observed for all junctions. This is in contrast to the typical $R_{J}(T)$ behavior for a junction with a nonmagnetic barrier, such as $\mathrm{Al}_{2} \mathrm{O}_{3}$, for which $\Phi$ is constant at all $\mathrm{T}$ and $\mathrm{R}_{\mathrm{J}}$ increases monotonically by $\sim 15 \%$ as $\mathrm{T}$ decreases [16].

The exchange splitting in this $2.5 \mathrm{~nm} \mathrm{EuO}$ barrier is quantitatively determined from $\mathrm{R}_{\mathrm{J}}(\mathrm{T})$ and current-voltage $(\mathrm{I}-\mathrm{V})$ measurements in the following way. First, by fitting the IV curve to the Brinkman-Dynes-Rowell (BDR) model [6] for tunneling between two metals through an insulating barrier, the average barrier height $\Phi_{0}$ and thickness $d$ were found. Beginning with the $\mathrm{I}-\mathrm{V}$ curve at $98 \mathrm{~K}$, at which $2 \Delta \mathrm{E}_{\mathrm{ex}}=0$, the BDR fit yielded $\Phi_{0}=0.92 \mathrm{eV}$ and $d=3.1 \mathrm{~nm}$. Then, holding the $\Phi_{0}$ and $d$ values constant, the $\mathrm{I}-\mathrm{V}$ curve at $6 \mathrm{~K}$ was fit by varying $\Delta \mathrm{E}_{\mathrm{ex}}$ such that $\Phi_{0}-\Delta \mathrm{E}_{\mathrm{ex}}=\Phi_{\uparrow}$ and $\Phi_{0}+\Delta \mathrm{E}_{\mathrm{ex}}=\Phi_{\downarrow}$ and the total 
current $\mathrm{I}=\mathrm{I}_{\uparrow}+\mathrm{I}_{\downarrow}$, where the spin-up(spin-down) current $\mathrm{I}_{\uparrow}\left(\mathrm{I}_{\downarrow}\right)$ corresponds to $\Phi_{\uparrow}\left(\Phi_{\downarrow}\right)$. This procedure yielded a $2 \Delta \mathrm{E}_{\mathrm{ex}}$ of $0.29 \mathrm{eV}$, which is quite large for this ultrathin $\mathrm{EuO}$ film, though reduced from the bulk value of $0.54 \mathrm{eV}$. Importantly, it is seen here that tunneling can be utilized to quantitatively determine the exchange splitting in a magnetic insulator, a first such demonstration of extending the principle of electron tunneling to study magnetism in ultrathin films, which is nontrivial, especially in systems as unstable as EuO.

Data analysis using the BDR model was performed for I-V curves in the bias range $150 \mathrm{mV}$ to $150 \mathrm{mV}$, which is consistent with the $\mathrm{R}_{\mathrm{J}}(\mathrm{T})$ curve at $\mathrm{V}=100 \mathrm{mV}$, shown again in Fig. 4b. Limiting the fit to this range ensured that $\Phi_{0}$ was not a function of bias $[6,17]$. Shown along with the measured $R_{J}(T)$ is a simulated $R_{J}(T)$ curve obtained by calculating $\mathrm{R}_{\mathrm{J}}$ using the $\mathrm{BDR}$ relation, with $\Phi_{0}=0.92 \mathrm{eV}$ and $d=3.1 \mathrm{eV}$, and from the $\mathrm{M}(\mathrm{T})$ curve in Fig. 2 for the $3 \mathrm{~nm} \mathrm{EuO} \mathrm{film.} \mathrm{Here,} \mathrm{the} \mathrm{assumption} \mathrm{was} \mathrm{that} 2 \Delta \mathrm{E}_{\text {ex }}$ varies with temperature as $\mathrm{M}(\mathrm{T})$, such that $\frac{2 \Delta \mathrm{E}_{\mathrm{ex}}(\mathrm{T})}{2 \Delta \mathrm{E}_{\mathrm{ex}}(\mathrm{T}=5 \mathrm{~K})}=\frac{\mathrm{M}(\mathrm{T})}{\mathrm{M}(\mathrm{T}=5 \mathrm{~K})}$. The good agreement between the measurement and the simulation nicely correlates the transport in the $\mathrm{EuO}$ barrier to the EuO magnetization.

Furthermore, knowing the $\mathrm{I}_{\uparrow}$ and $\mathrm{I}_{\downarrow}$ values from the BDR fit, the spin polarization of the tunnel current is calculated from $\mathrm{P}=\left(\mathrm{I}_{\uparrow}-\mathrm{I}_{\downarrow}\right) /\left(\mathrm{I}_{\uparrow}+\mathrm{I}_{\downarrow}\right)$, shown in Fig. 4b. This resulted in nearly total polarization of $98 \%$ for $2 \Delta \mathrm{E}_{\mathrm{ex}}=0.29 \mathrm{eV}\left(\mathrm{P}=0\right.$ for $\left.2 \Delta \mathrm{E}_{\mathrm{ex}}=0\right)$. Thus, the splitting in this ultrathin $\mathrm{EuO}$ barrier is large enough to produce a near fully spin-polarized current, demonstrating its potential as an ideal spin source and injector. The same analysis carried out on tunneling measurements for a $1.5 \mathrm{~nm} \mathrm{EuO}$ barrier from the same sample set yielded $\Phi_{0}=0.35 \mathrm{eV}, 2 \Delta \mathrm{E}_{\mathrm{ex}}=0.10 \mathrm{eV}$ and $\mathrm{P}=97 \%$. Despite the smaller $2 \Delta \mathrm{E}_{\mathrm{ex}}$ for the thinner barrier, in line with the lower $\mathrm{T}_{\mathrm{C}}$, a large polarization is still produced because the splitting remains substantial relative to the lower average barrier height of the thinner barrier.

It should be noted that this analysis does not take into account any spin scattering events that could cause a loss of $\mathrm{P}$ during tunneling, such as interfacial impurities, defect states or excitation of magnons at high bias. A spin detector, such as a superconducting electrode used in the Meservey-Tedrow technique [18], or a ferromagnetic electrode used in a quasi-magnetic tunnel junction structure [19], would directly probe P.

Figure $4 \mathrm{a}$ also shows $\mathrm{R}_{\mathrm{J}}(\mathrm{T})$ at higher applied $\mathrm{V}$. The amount of decrease in $\mathrm{R}_{\mathrm{J}}$ (given by $\frac{\Delta R}{R}=\frac{R_{J, \max }-R_{J}(6 K)}{R_{J, \max }}$, where $R_{J, \max }$ is the maximum $R_{J}$ ) is larger at $400 \mathrm{mV}$ and $800 \mathrm{mV}$. 
High applied V can drastically deform the potential barrier, resulting in Fowler-Nordheim (FN) tunneling. The larger $\Delta \mathrm{R} / \mathrm{R}$ at higher bias can be attributed to $\mathrm{FN}$ tunneling into the spin-up band of $\mathrm{EuO}$ - an effect that is also known to produce high $\mathrm{P}$ at high $\mathrm{V}$ in EuS barriers [20,21]. Observation of this large $\Delta R / R$ at high bias signifies that the spin-filter effect persists even at high bias, which is relevant for operating a spintronic device. When even higher $\mathrm{V}$ is applied, the barrier height is so low (modified by bias) that change in $\Phi_{0}$ by exchange splitting does not make a significant change in tunneling probability, so that $\mathrm{P}$ would decrease as observed in our earlier work [20].

In conclusion, this fundamental study of the magnetic properties of high-quality, ultrathin $\mathrm{EuO}$ films revealed a reduction in Curie temperature from bulk value as thickness is reduced to a few monolayers. We have utilized the spin-filter capability of $\mathrm{EuO}$ barriers to quantitatively determine the amount of exchange splitting in the conduction band. Such an approach can be extended to other magnetic semiconductors and insulators. The spin-filter effect displayed here shows the potential of $\mathrm{EuO}$ to generate a fully spin-polarized current for spin injection.

This work is funded by NSF grants, ONR grant, and partially by the KIST-MIT program. Some of us (EN, JH, JD, ML, and YI) were supported by the Office of Naval Research under grant N00014-03-1-0692, by the DOE under DE-AC06-76RL01830, and by NSF under DMR0210915. The ALS is supported by the U.S. Department of Energy under Contract No. DEAC02-05CH11231.

[1] P. Wachter, in Handbook on the Physics and Chemistry of Rare Earths, edited by K. Schneider and L. Eyring (North-Holland Publishing Co., New York, 1979), vol. 2, pp. 507-574.

[2] J. Moodera, X. Hao, G. Gibson, and R. Meservey, Phys. Rev. Lett. 61, 637 (1988); X. Hao, J. Moodera, and R. Meservey, Phys. Rev. B 42, 8235 (1990).

[3] J. Moodera, R. Meservey, and X. Hao, Phys. Rev. Lett. 70, 853 (1993).

[4] T. S. Santos and J. S. Moodera, Phys. Rev. B 69, 241203(R) (2004).

[5] G. Busch, P. Junod, and P. Wachter, Phys. Lett. 12, 11 (1964).

[6] W. F. Brinkman, R. C. Dynes, and J. M. Rowell, J. Appl. Phys. 41, 1915 (1970).

[7] J. S. Moodera, T. S. Santos, and T. Nagahama, J. Phys.: Cond. Matt. 19, 165202 (2007).

[8] R. Schiller and W. Nolting, Phys. Rev. Lett. 86, 3847 (2001); R. Schiller, W. Müller, and 
W. Nolting, Phys. Rev. B 64, 134409 (2001).

[9] in The Oxide Handbook, edited by G. V. Samsonov (IFI/Plenum, New York, 1982), 2nd ed.

[10] E. Negusse, J. Holroyd, M. Liberati, J. Dvorak, Y. U. Idzerda, T. S. Santos, J. S. Moodera, and E. Arenholz, J. Appl. Phys. 99, 08E507 (2006).

[11] B. T. Thole, G. van der Laan, J. C. Fuggle, G. A. Sawatzky, R. C. Karnatak, and J.-M. Esteva, Phys. Rev. B 32, 5107 (1985).

[12] C. Wittkop, W. Schülke, and F. M. F. de Groot, Phys. Rev. B 61, 7176 (2000).

[13] H. Ott, S. J. Heise, R. Sutarto, Z. Hu, C. F. Chang, H. H. Hsieh, H.-J. Lin, C. T. Chen, and L. H. Tjeng, Phys. Rev. B 73, 094407 (2006).

[14] F. Leuenberger, A. Parge, W. Felsch, K. Fauth, and M. Hessler, Phys. Rev. B 72, 014427 (2005).

[15] $\mathrm{Mg}$ was deposited at the bottom $\mathrm{Al} / \mathrm{EuO}$ interface in half of the junctions, in an effort to minimize $\mathrm{Al}_{2} \mathrm{O}_{3}$ formation, though whether $\mathrm{Mg}$ was present or not, did not have any appreciable effect on the $\mathrm{R}_{\mathrm{J}}(\mathrm{T})$ behavior.

[16] J. S. Moodera and G. Mathon, J. Magn. Magn. Mater. 200, 248 (1999).

[17] C. W. Miller, Z.-P. Li, I. K. Schuller, R. W. Dave, J. M. Slaughter, and J. Åkerman, Phys. Rev. B 74, 212404 (2006).

[18] R. Meservey and P. M. Tedrow, Phys. Rep. 238, 173 (1994).

[19] P. LeClair, J. K. Ha, H. J. M. Swagten, J. T. Kohlhepp, C. H. van de Vin, and W. J. M. de Jonge, Appl. Phys. Lett. 80, 625 (2002).

[20] T. Nagahama, T. S. Santos, and J. S. Moodera, Phys. Rev. Lett. 99, 016602 (2007).

[21] A. Saffarzadeh, J. Magn. Magn. Mater. 269, 327 (2004). 